\title{
Immunogenicity after the first dose of the BNT162b2 mRNA Covid-19 vaccine: real-world evidence from Greek healthcare workers
}

\author{
Konstantina Kontopoulou', Alexandra Ainatzoglou'2, Athina Ifantidou', Christos T. Nakas ${ }^{3,4}$, Georgia Gkounti $^{5}$, \\ Vasilios Adamopoulos' ${ }^{1}$ Nikitas Papadopoulos ${ }^{1}$ and Georgios Papazisis²,6,*
}

\begin{abstract}
Real-world data regarding the effectiveness, safety and immunogenicity of the Pfizer-BioNTech BNT162b2 mRNA vaccine are accumulating in the literature, suggesting that this vaccine generates high titres of S1-binding IgG antibodies that exhibit potent virus neutralization capacity. This is the first phase IV immunogenicity study to recruit a large number of Greek healthcare workers $(n=425)$ including 63 previously-infected subjects. We measured titres of neutralizing IgGs against the receptor-binding domain of the S1 subunit of the spike protein of SARS-CoV-2 14 days post-immunization with the first dose, employing the SARS-CoV-2 IgG II Quant assay. A total of $92.24 \%$ of our study cohort received a positive assay outcome and titres varied with age. Post-hoc analysis revealed that although titres did not significantly differ among participants aged 20-49years, a significant decline was marked in the age group of 50-59 years, which was further accentuated in subjects aged over 60 . Antibody titres escalated significantly among the previously-infected, indicating the potential booster effect of the first dose in that group.
\end{abstract}

\section{DATA SUMMARY}

The authors confirm all supporting data, code and protocols have been provided within the article or through supplementary data files.

\section{INTRODUCTION}

In December 2020, the Pfizer-BioNTech BNT162b2 mRNA vaccine received EMA authorization for emergency use to mitigate the multifaceted impact of the Covid-19 pandemic. Vaccine effectiveness post-immunization with the second dose was measured at $95 \%$ in phase III trials [1] and $92 \%$ as of latest phase IV findings [2]. Regarding its immunogenic profile, the BNT162b2 vaccine generated high titres of S1-binding IgG that exhibited potent virus neutralizing capacity in phase II trials $[3,4]$. These data were just recently confirmed by the first phase IV immunogenicity study [5], reporting $92 \%$ efficiency of the first dose in inducing a robust humoral immune response, an effect that was further accentuated among previously SARS$\mathrm{CoV}-2$-infected subjects. These findings, along with accumulating evidence [6,7], raise a conundrum regarding the necessity of a booster dose among seropositive individuals. Data provided by our study can be harnessed to address this fervent issue, as this is the first phase IV study to collect immunogenicity data of such a large number of previously-infected vaccinated participants $(n=63)$.

The biotechnology of this lipid nanoparticle (LNP)encapsulated messenger RNA (mRNA) vaccine conveys the genetic information of the viral immunogen to host antigen presenting cells, sparking a robust immune response [8]. The mRNA molecule is briefly expressed and not incorporated in the genome, undergoing rapid eradication by physiological cellular routes. The manufacture of mRNA vaccines takes place in vitro through transcription of DNA templates, without the need for

Received 10 March 2021; Accepted 21 May 2021; Published 16 August 2021

Author affiliations: 'Department of Microbiology, 'G. Gennimatas' General Hospital, Thessaloniki, Greece; ${ }^{2}$ Department of Clinical Pharmacology, School of Medicine, Aristotle University of Thessaloniki, Thessaloniki, Greece; ${ }^{3}$ Laboratory of Biometry, University of Thessaly, Phytokou Str.38446, Nea Ionia/Volos, Magnesia, Greece; ‘University Institute of Clinical Chemistry, Inselspital, Bern University Hospital, University of Bern, Bern, Switzerland; ${ }^{5}$ Department of Cardiology, 'G. Gennimatas' General Hospital, Thessaloniki, Greece; ${ }^{6}$ Clinical Trials Unit, Special Unit for Biomedical Research and Education, School of Medicine, Aristotle University of Thessaloniki, Thessaloniki, Greece.

*Correspondence: Georgios Papazisis, papazisg@auth.gr

Keywords: vaccine; Pfizer-BioNTech BNT162b2 mRNA; SARS-CoV-2; immunogenicity; healthcare workers; Greece.

Abbreviations: Covid-19, coronavirus infectious disease 2019; EMA, European Medicines Agency; LNP, lipid nanoparticle; mRNA, messenger

riboneucleic acid; SARS-CoV-2, severe acute respiratory syndrome coronavirus.

Our study has been displayed as a preprint in the SSRN repository under the access number 3786138.

001387 (c) 2021 The Authors 
animal-derived supplies [9]. This is a swift, well-established and adjustable process, constituting this biotechnology highly eligible for fast deliverables and constant vaccine supply, both of which are imperative during any pandemic [10]. With respect to the BNT162b2, administration of this LNP-encapsulated nucleoside-modified mRNA follows a regimen of two $30 \mu \mathrm{g}$ doses intramuscularly injected 21 days apart. When entered into the recipient, this mRNA formula encodes the entire SARSCoV-2 stabilized perfusion spike protein [10].

Overall, our study aims to enhance insight into the humoral immunity and neutralizing antibody generation elicited 14 days post-immunization with the first dose of BNT162b2mRNA Covid-19 vaccine in real-world settings.

\section{METHODS}

Our study population encompassed 425 Greek healthcare workers, 63 of whom were previously-infected convalescent subjects with PCR-confirmed SARS-CoV-2 infection one to 4.5 months prior to vaccination with the Pfizer-BioNTech Covid-19 BNT162b2 vaccine (Pfizer, Inc; Philadelphia, Pennsylvania). Administration of the first dose occurred in the 'G. Gennimatas' General Hospital of Thessaloniki from 4 January 2021 through 7 January 2021 through intramuscular injection. Blood withdrawal and serum isolation were performed 14 days post-immunization. Subsequently, the SARS-CoV-2 IgG II Quant assay was employed on serum samples to monitor titres

\section{Impact Statement}

This is the first phase IV immunogenicity study to recruit such a large number of previously-infected convalescent subjects ( $n=63$ ), contributing to the resolution of ongoing conundrum regarding the redundancy of second dose administration to previously-infected individuals.

of neutralizing IgGs against the receptor-binding domain (RBD) of the S1 subunit of the spike protein of SARS-CoV-2, using the Architect System (Abbott, Sligo, Ireland). This is an automated two-step chemiluminescent micro-particle assay characterized by $99.357 \%$ sensitivity and $99.6 \%$ specificity [11]. Based on representative data for the limit of quantitation and detection, the ranges over which results can be reported are 6.8-80000 $\mathrm{AU} \mathrm{ml}{ }^{-1}$. Values of RBD-IgG concentration obtained by this technique were converted to IgG geometric mean concentrations (GMCs).

Stata 16.1 (Stata Corp. LLC, College Station, TX) was used for data analysis. Descriptive statistics included geometric means of concentration (GMC) of RBD-specific IgG $\left.(\mathrm{AU} \mathrm{ml})^{-1}\right)$ and the corresponding $95 \%$ confidence intervals $(95 \% \mathrm{CI})$ of each studied group classified by gender, age and history of previous SARS-CoV-2 infection. Independent samples $t$-tests were used

Table 1. Geometric means of concentration (GMC) of Anti SARS-CoV-2 Spike IgG antibodies 14 days post-immunization with the first dose of the BNT162b2 vaccine

\begin{tabular}{|c|c|c|c|c|c|}
\hline & & $\mathbf{n}$ & $\%$ & $\operatorname{IgG~GMC~}\left(\mathrm{AU} \mathrm{ml} \mathbf{l}^{-1}\right)$ & $95 \% \mathrm{CI}$ \\
\hline \multirow[t]{2}{*}{ Sex } & Female & 256 & 60 & 581.01 & $(462.41,730.02)$ \\
\hline & Male & 169 & 40 & 448.87 & $(322.88,624.03)$ \\
\hline \multirow{4}{*}{ Age } & $30-$ & 69 & 16 & 1552.00 & $(1054.22,2284.82)$ \\
\hline & $40-$ & 123 & 29 & 674.35 & $(488.95,930.06)$ \\
\hline & $50-$ & 166 & 39 & 357.70 & $(261.23,489.79)$ \\
\hline & $60-$ & 51 & 12 & 155.80 & $(94.07,258.04)$ \\
\hline Prior infection ${ }^{*}$ & No & 362 & 85 & 278.24 & $(242.66,319.05)$ \\
\hline \multirow[t]{2}{*}{$\mathrm{IgG}>50 \mathrm{AU} \mathrm{ml}{ }^{-1}$} & Yes & 392 & 92.24 & 702.89 & $(590.70,836.39)$ \\
\hline & No & 33 & 7.76 & 16.14 & $(10.73,24.26)$ \\
\hline Total & & 425 & & 524.35 & $(433.83,633.75)$ \\
\hline
\end{tabular}

*PCR-confirmed SARS-CoV-2 infection 1 to 4.5 months prior to immunization date. 


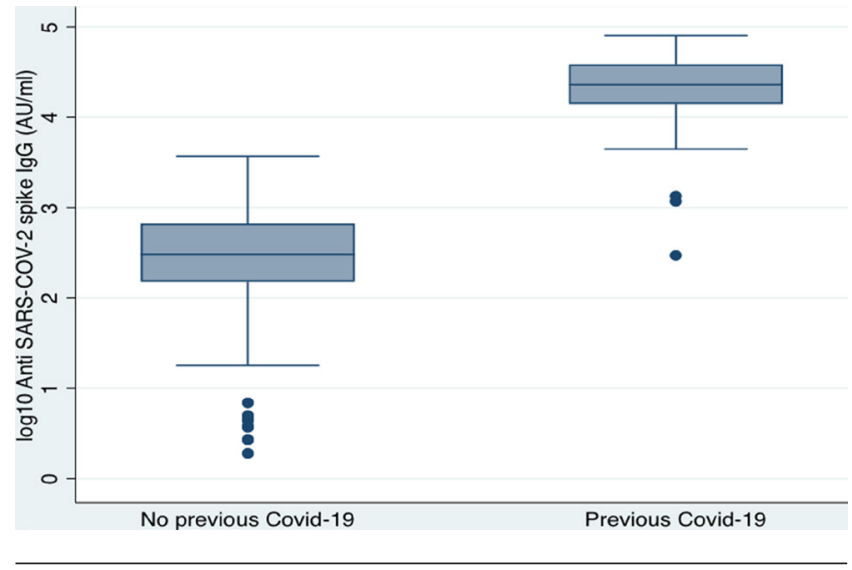

Fig. 1. Highly significant difference between participants with and without prior SARS-CoV-2 infection $(P<0.001)$.

to assess differences in $\log 10$ IgG levels among gender- and SARS-CoV-2 infection history groups. One-way ANOVA with Scheffe correction for multiplicity was employed for the comparison of age groups. ROC curve analysis was used to assess IgG levels in participants with history of SARS-CoV-2 infection (AUC $=0.988 ; 95 \%$ CI: $0.972,1.000$ ) and $P$-values less than 0.05 were considered statistically significant.

\section{RESULTS}

In this study, the 425 healthcare workers recruited included subjects with both a positive $(n=63)$ and a negative history $(n=362)$ of SARS-CoV-2 infection. The mean age of participants was 48 , ranging between 21 to 67 years, all participants were Caucasian, while women accounted for $60 \%$ of the study population.

Regarding immunogenicity, 392 participants received a positive outcome in the IgG assay conducted 14 days after the first dose, comprising $92.2 \%$ of the total study population. In this group,

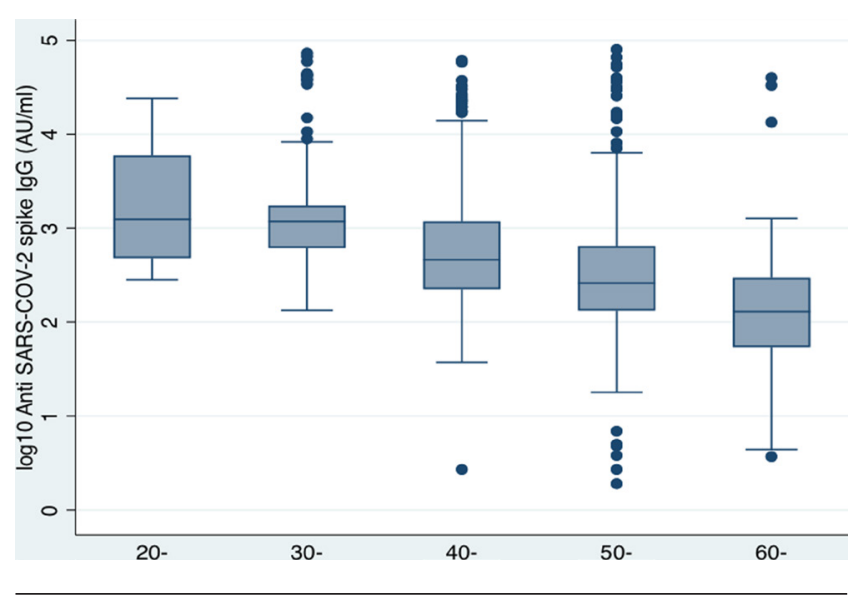

Fig. 2. Highly significant difference between age groups $(P<0.001)$. Scheffe post-hoc testing results in significant differences between 20-49,50-59 and 60+ years old.

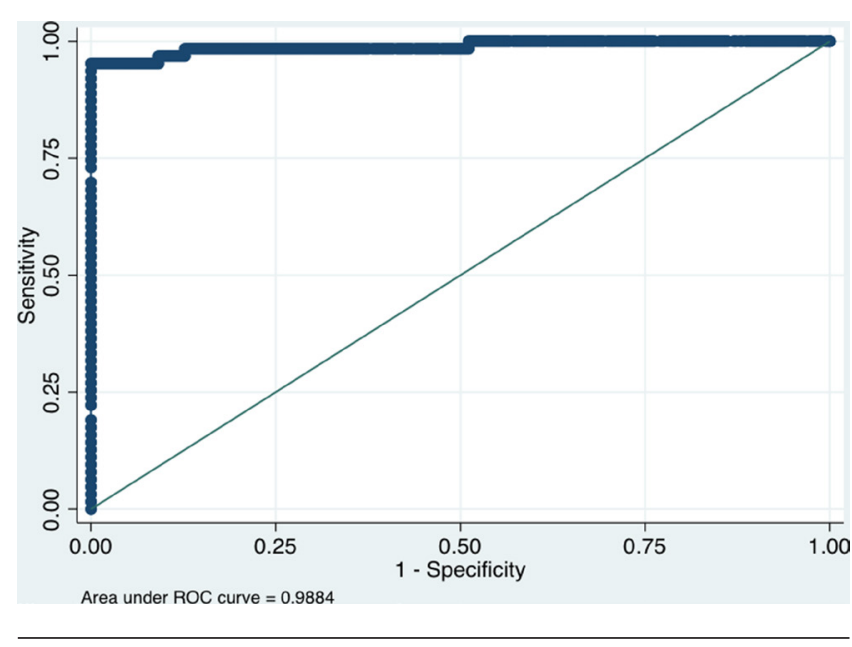

Fig. 3. ROC curve analysis for the assessment of IgG levels for the diagnosis of previous SARS-CoV-2 infection (AUC=0.988; $95 \% \mathrm{Cl}$ : $0.972,1.000$ ) revealing a cut-off score of $>=4439.4$ (sensitivity: $95.24 \%$, specificity: 100\%).

IgG concentration values ranged between 54-80000 $\mathrm{AU} \mathrm{ml}^{-1}$, with a GMC of $702.89 \mathrm{AU} \mathrm{ml}^{-1}$. In the remaining 33 participants (7.76\%) who received a negative assay outcome, IgG concentration was lower than $50 \mathrm{AU} \mathrm{ml}^{-1}$, with a GMC of $16.14 \mathrm{AU} \mathrm{ml}^{-1}$. (Table 1)

In all 63 previously-infected participants, a positive assay outcome was obtained, with IgG concentration values spanning between $295.5-80,000 \mathrm{AU} \mathrm{ml}^{-1}$. In the previously-infected group, the GMC of RBD-IgG was estimated at $19993.61 \mathrm{AU}$ $\mathrm{ml}^{-1}$, compared to $278.24 \mathrm{AU} \mathrm{ml}^{-1}$ recorded in the uninfected group. (Table 1) Expectedly, there was a large and statistically significant difference in antibody GMCs between the previouslyinfected and the uninfected group $(P<0.001)$. (Fig. 1$)$

Antibody titres fluctuated in the various age groups (One-way ANOVA $P<0.001)$. Post-hoc testing revealed that although titres did not significantly differ among participants aged $20-49$ years, a significant decline was marked in the age group of 50-59 years, which was further accentuated in subjects aged over 60. (Fig. 2)

Through ROC curve analysis, an optimal IgG cut-off score of $\geq 4439.4 \mathrm{AU} \mathrm{ml}^{-1}$ was found to be explicitly diagnostic of previous SARS-CoV-2 infection (AUC $=0.988$; 95\% CI: 0.972, 1.000 ) resulting in $95.24 \%$ sensitivity and $100 \%$ specificity via a maximum of the Youden index criterion. (Fig. 3)

Regarding reactogenicity, neither serious adverse events (AEs), nor withdrawals due to related AEs were observed in this study. In addition, none of our study participants declared a yellow card submission, evidence confirming the absence of serious AEs. The most common local side effect reported was mild to moderate pain at the injection site, while the most common systemic $\mathrm{AE}$ was fatigue followed by myalgias, headache and chills. Participants with a positive history of SARS-CoV-2 infection seemed to experience more local and systemic reactions, including fever. 


\section{DISCUSSION}

Overall, real-world evidence provided by our study further verifies the immunogenic capacity of the first dose of the BNT162b2 mRNA Covid-19 vaccine, also confirming its efficiency in inducing a rapid and robust immune response in $92.2 \%$ of our study participants. Data presented in our study focuses on the humoral branch of the adaptive immune response generated by the first dose and should not be interpreted as the sole immunogenic effect of the BNT162b2 on its recipients. However, levels of S1-binding IgG exhibited a direct correlation with the intensity of S1-specific CD4+ and CD8+ Tcell responses in a phase II RCT [4], reflecting the notion of intramolecular synergy and adding to the value of the high IgG GMCs reported in our study.

Our data are consistent with those provided by the first phase IV immunogenicity study [5], that documented neutralizing antibody generation 21 days post-injection of the first dose of the BNT162b2 vaccine in 514 Israeli healthcare workers. As mentioned in that study, $92 \%$ of participants had a positive SARS-CoV-2 IgG assay, titres seemed to decline with increasing age, while appearing significantly elevated among all 17 previously-infected participants [5]. These findings, along with those of other studies [7], question the necessity of a second dose among subjects with a positive history of SARS-CoV-2 infection, given the booster effect of the first dose observed in this group. This remark has further been supported by immunogenicity data on the second dose of the vaccine lately provided by a small-scale study, reporting a muted effect of the second dose among previously-infected individuals, in contrast to its booster effect in the uninfected [6].

\section{CONCLUSION}

Real-world evidence reported in this study confirms the robust immunogenic effect of the first dose of the BNT162b2 mRNA Covid-19 vaccine, which was found significantly pronounced among previously-infected subjects, indicating the potential booster effect of the first dose in that group.

Funding information

This work received no specific grant from any funding agency.
Conflicts of interest

The authors declare that there are no conflicts of interest.

Ethical statement

The study protocol was reviewed and approved by the Scientific Committee of 'G. Gennimatas' General Hospital (protocol number:1/13.1.2021). All subjects agreed to voluntarily participate in the study. The trial is registered on the International Standard Randomized Controlled Trial Number registry (study ID: ISRCTN61884303).

\section{References}

1. Polack FP, Thomas SJ, Kitchin N, Absalon J, Gurtman A, et al. Safety and efficacy of the BNT162b2 mRNA Covid-19 vaccine. N Engl J Med 2020;383:2603-2615.

2. Dagan N, Barda N, Kepten E, Miron O, Perchik S, et al. BNT162b2 mRNA Covid-19 Vaccine in a Nationwide Mass Vaccination Setting. N Engl J Med 2021;384:1412-1423.

3. Walsh EE, Frenck RW, Falsey AR, Kitchin N, Absalon J, et al. Safety and Immunogenicity of Two RNA-Based Covid-19 Vaccine Candidates. N Engl J Med 2020;383:2439-2450.

4. Sahin U, Muik A, Vogler I, Derhovanessian E, Kranz LM, et al. BNT162b2 induces SARS-CoV-2-neutralising antibodies and T cells in humans. medRxiv 2020

5. Abu Jabal K, Ben-Amram H, Beiruti K, Batheesh Y, Sussan C, et al. Impact of age, ethnicity, sex and prior infection status on immunogenicity following a single dose of the BNT162b2 mRNA COVID-19 vaccine: real-world evidence from healthcare workers, Israel, December 2020 to January 2021. Eurosurveillance 2021;26:2100096

6. Samanovic MI, Cornelius AR, Wilson JP, Karmacharya T, Gray-Gaillard SL, et al. Poor antigen-specific responses to the second BNT162b2 mRNA vaccine dose in SARS-CoV-2-experienced individuals. medRxiv [Internet] 2021.

7. Krammer F, Srivastava K, Team P, Simon V, Alshammary H, et al. Robust spike antibody responses and increased reactogenicity in seropositive individuals after a 1 single dose of SARS-COV-2 mRNA vaccine 2 3. medRXiv 2015.

8. Pardi N, Hogan MJ, Naradikian MS, Parkhouse K, Cain DW, et al. Nucleoside-modified mRNA vaccines induce potent $\mathrm{T}$ follicular helper and germinal center B cell responses. J Exp Med 2018;215:1571.

9. Rauch S, Jasny E, Schmidt KE, Petsch B. New vaccine technologies to combat outbreak situations. Front Immunol 2018;9:1963.

10. Wrapp D, Wang N, Corbett KS, Goldsmith JA, Hsieh CL, et al. Cryo-EM structure of the 2019-nCoV spike in the prefusion conformation. bioRxiv 2020

11. SARS-CoV-2 Immunoassay. Abbott core laboratory (Internet). 2021. https://www.corelaboratory.abbott/int/en/offerings/segments/infectious-disease/sars-cov-2

\section{Five reasons to publish your next article with a Microbiology Society journal \\ 1. The Microbiology Society is a not-for-profit organization. \\ 2. We offer fast and rigorous peer review - average time to first decision is 4-6 weeks. \\ 3. Our journals have a global readership with subscriptions held in research institutions around the world. \\ 4. $80 \%$ of our authors rate our submission process as 'excellent' or 'very good'. \\ 5. Your article will be published on an interactive journal platform with advanced metrics.}

Find out more and submit your article at microbiologyresearch.org. 